\title{
Les méthodes adaptatives : quand et comment les utiliser dans les essais cliniques?
}

\author{
Raphaël Porcher ${ }^{1}$, Brigitte Lecocq ${ }^{2}$, Muriel Vray ${ }^{3}$ et les participants de la Table Ronde $N^{\circ} 2$ de Giens XXVI \\ 1 Université Paris Diderot, Hôpital Saint-Louis, Paris, France \\ 2 Novartis Pharma S.A.S., Rueil Malmaison, France \\ 3 Institut Pasteur, Paris, France
}

Texte reçu le 8 avril 2011 ; accepté le 16 mai 2011

\begin{abstract}
Mots clés :
analyse intermédiaire ; essais adaptatifs ; modification du plan d'expérience ; sélection de traitement

Résumé - On définit les schémas adaptatifs pour les essais cliniques comme les schémas qui utilisent les données accumulées dans l'essai pour en modifier éventuellement certains aspects, sans compromettre la validité et l'intégrité de l'essai. Par rapport aux essais plus traditionnels, les schémas adaptatifs permettent en théorie de produire la même information, mais de manière plus efficiente. Les avantages et les limites de ce type de schémas ainsi que le poids des contraintes, notamment logistiques, liés à leur utilisation, diffèrent selon que l'essai est un essai exploratoire ou un essai confirmatoire, en vue d'un enregistrement. L'un des éléments importants pour garantir l'intégrité de l'essai est le recours à un comité indépendant pour déterminer les adaptations du plan d'expérience en cours d'essai. Les méthodes adaptatives pour les essais cliniques sont attrayantes et peuvent être acceptées par les autorités compétentes. Elles nécessitent cependant de bien mesurer à l'avance les contraintes qu'elles imposent.
\end{abstract}

\section{Introduction}

Le concept d'essai adaptatif a récemment suscité beaucoup d'intérêt à la fois dans la littérature biostatistique (numéros spéciaux de Journal of Biopharmaceutical Statistics en 2005 et de Biometrical Journal en 2006, revues de la littérature, ${ }^{[1-5]}$ mais aussi au niveau des agences de régulation. ${ }^{[6-8]}$

Selon les auteurs, ce concept peut regrouper des notions très différentes. Une taxonomie assez détaillée a été proposée dans le cadre de réflexions menées par le groupe PhRMA, ${ }^{[9]}$ et un schéma adaptatif est maintenant généralement défini comme un plan d'expérience dans lequel les données accumulées au cours de l'essai sont utilisées pour éventuellement en modifier certains aspects, sans en compromettre la validité et l'intégrité. C'est cette définition qui est reprise dans le projet de guide de la Food and drug administration (FDA). ${ }^{[8]}$ Le terme validité de l'essai signifie que l'inférence statistique doit être correcte en termes d'estimation et de tests (en particulier le contrôle de l'erreur de type I ou risque de première espèce), qu'une cohérence doit être maintenue entre les différentes étapes de l'essai et que les biais opérationnels doivent

\footnotetext{
* Pour la liste des participants, voir en fin d'article
}

être minimisés. L'intégrité se rapporte à l'acceptabilité des résultats par la communauté scientifique au sens large, notamment par les autorités compétentes. Cela passe notamment par une planification à l'avance des adaptations autant que possible, et par le maintien de la confidentialité des données au cours de l'essai. Les possibilités d'adaptation du plan d'expérience sont très larges, et on peut citer comme cible d'adaptations possibles les critères d'éligibilité dans l'essai, les règles de randomisation (modifier le ratio d'allocation de chaque traitement), la taille de l'échantillon (y compris un arrêt précoce de l'essai), les traitements (dose, durée, ...), les critères d'évaluation (y compris le critère principal, par exemple dans le cas d'un critère composite), la méthode d'analyse ... Sans entrer dans la taxonomie détaillée citée plus haut, on peut cependant résumer les différents types de schémas adaptatifs comme présenté dans le tableau I.

D'après la définition retenue, un essai dont le plan d'expérience est modifié exclusivement sur des données extérieures à l'essai n'utilise pas un schéma adaptatif. En revanche, un essai séquentiel par groupe où un arrêt précoce pour futilité ou efficacité est prévu après une ou plusieurs analyses intermédiaires entre dans le cadre des schémas adaptatifs, (pour certains auteurs, 
Tableau I. Principaux schémas adaptatifs.

\begin{tabular}{ll}
\hline Type d'essai adaptatif & Adaptations \\
\hline $\begin{array}{l}\text { Essai séquentiel par groupe } \\
\text { Réévaluation du nombre de sujets en aveugle } \\
\text { (ré-estimation de la variance, ...) }\end{array}$ & $\begin{array}{l}\text { Arrêt précoce de l'étude } \\
\text { Modification de la taille d'échantillon }\end{array}$ \\
$\begin{array}{l}\text { Phase 1 escalade de doses CRM modifiée } \\
\text { Phase 1-2 intégrés }\end{array}$ & $\begin{array}{l}\text { Choix de la prochaine dose } \\
\text { Choix de la prochaine dose }\end{array}$ \\
$\begin{array}{l}\text { Réévaluation du nombre de sujets } \\
\text { (sans aveugle, sur des critères d'efficacité) }\end{array}$ & $\begin{array}{l}\text { Changement du ratio de randomisation } \\
\text { Enrichissement de la population }\end{array}$ \\
& Augmentation de la taille d'échantillon \\
Essais combinés phases 2-3 (seamless phase 2-3) & $\begin{array}{l}\text { Modification des critères d'inclusion, } \\
\text { de la population d'analyse, sous-groupes }\end{array}$ \\
\hline
\end{tabular}

CRM : Continual Reassessment Method

ces essais où le côté adaptatif est entièrement pré-spécifié ne sont cependant pas complètement adaptatifs). ${ }^{[10]}$ Parmi les schémas adaptatifs on trouve aussi les méthodes de réévaluation du nombre de sujets nécessaire, réalisées en aveugle sur données agrégées. Dans ce dernier cas, un paramètre de nuisance, comme la variance du critère d'évaluation, est estimé en cours d'étude pour réviser la valeur utilisée dans le calcul du nombre de sujets nécessaire lors de la planification et modifier si besoin ce nombre de sujets pour maintenir la puissance de l'étude. Ces deux types de schémas adaptatifs sont utilisés depuis longtemps, les méthodes statistiques sont bien développées, et leur utilisation ne semble plus poser de questions particulières vis-à-vis des agences de régulation. Les schémas adaptatifs ont donc été séparés entre ceux qui étaient bien maîtrisés ou bien connus (well understood) et les autres (less well understood). ${ }^{[8]}$ Les travaux de la Table Ronde ont plus spécifiquement porté sur ces schémas moins bien connus, laissant de côté par exemple les essais séquentiels par groupe.

En regard des schémas traditionnels où l'ensemble du plan d'expérience est fixé avant le début de l'essai, les schémas adaptatifs peuvent permettre d'obtenir la même information avec une meilleure efficacité, c'est-à-dire dans un délai plus court ou avec un nombre de sujets moindre. Leur flexibilité permet aussi d'augmenter la probabilité d'atteindre les objectifs de l'essai ou d'améliorer la connaissance de l'effet d'un traitement, en conduisant par exemple à une estimation plus précise de la relation dose-effet ou du taux de succès à la dose retenue en traitant plus de patients à cette dose. Cependant, l'utilisation de ce type de schéma n'est pas exempte de contraintes, parfois très lourdes, que nous nous sommes attachés à résumer dans la suite, et leur utilisation peut s'avérer risquée pour le promoteur d'un essai. Le compromis entre les avantages potentiels et le poids de ces contraintes et des risques est différent selon que l'essai est un essai exploratoire ou un essai confirmatoire, en vue d'un enregistrement.

Dans la suite, nous présenterons d'abord brièvement quelques concepts méthodologiques importants dans les schémas adaptatifs. Puis nous décrirons les types de schémas qui peuvent être utilisés pour des essais exploratoires au chapitre 3 et confirmatoires au chapitre 4, avec leurs avantages et leurs inconvénients. Le chapitre 5 décrira le rôle des différents intervenants et en particulier du comité indépendant. Les contraintes imposées par les schémas adaptatifs et les risques que leur utilisation peut engendrer seront enfin détaillés, avant une courte conclusion.

\section{Concepts de base}

Les concepts qui sous-tendent les méthodes adaptatives et les approches statistiques utilisées sont assez différents selon le type d'essai adaptatif. La définition d'un essai adaptatif présuppose cependant une conduite séquentielle de l'essai, avec une ou plusieurs analyses intermédiaires, afin de pouvoir adapter le plan d'expérience en fonction des données observées. Cette notion est donc centrale et commune à tout type d'essai adaptatif, qui peut être vu comme un essai séquentiel auquel on ajoute la possibilité d'adaptation. Le schéma adaptatif correspond donc à une extension du rôle de l'analyse intermédiaire. La définition même de l'analyse intermédiaire est aussi étendue dans le cas des schémas adaptatifs par rapport à celle usuellement retenue, c'est-à-dire une analyse dont l'objectif est de comparer les bras de traitement en termes d'efficacité ou de sécurité. ${ }^{[1]}$ Pour un schéma adaptatif, toute analyse de données de l'essai alors qu'il est en cours est considérée comme analyse intermédiaire, quelles que soient les données examinées ou l'objectif de cet examen. Le point crucial est alors de savoir si cette analyse a été faite complètement à l'insu du traitement ou non.

L'objectif de cet article n'est pas d'entrer dans le détail de toutes les méthodes statistiques utilisées dans les essais adaptatifs, et nous renvoyons le lecteur désireux d'approfondir ce point à des revues récentes de la littérature. ${ }^{[3,4]}$ Il nous a cependant paru important de résumer ici quelques concepts clé que l'on peut retrouver dans des essais adaptatifs. 


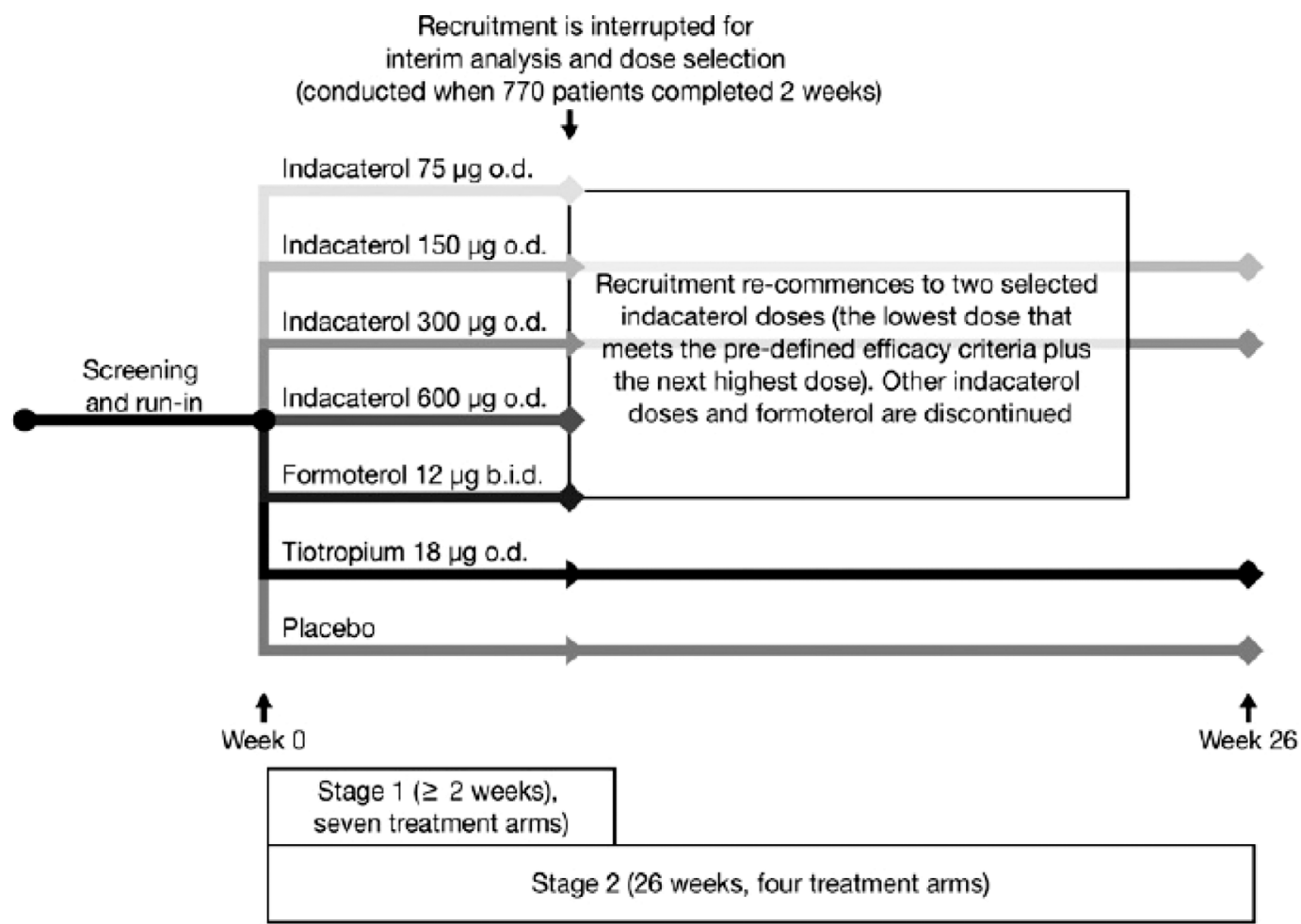

Fig. 1. Schéma d'un essai de phase 2-3 combinées (d'après Donohue et al. 2010, avec l'aimable autorisation de l'American Thoracic Society). [29]

\subsection{Contrôle de l'erreur de type I et principe d'invariance conditionnelle}

Pour les essais confirmatoires, un contrôle rigoureux du risque d'erreur de type I est nécessaire, afin de ne pas mettre en question la validité des conclusions. Tout essai sans contrôle strict de l'erreur de type I est donc considéré comme exploratoire. En revanche, malgré un contrôle de l'erreur de type I, de trop nombreuses adaptations, une taille d'échantillon insuffisante ou de trop nombreux critères d'évaluation induiront une certaine incertitude quant à la validité de l'essai.

Afin de contrôler le risque d'erreur de type I, les schémas adaptatifs répondent à un principe d'invariance conditionnelle. ${ }^{[12]}$ Supposons qu'en l'absence de toute modification du plan d'expérience le test final puisse s'exprimer en termes d'une combinaison de statistiques de test calculées aux différentes étapes de l'essai (par exemple sur les données recueillies entre les différentes analyses intermédiaires) et que la règle de décision sur cette statistique de test garantisse un risque d'erreur de type I $\alpha$. Alors, toute modification du plan d'expérience qui préserve la distribution sous l'hypothèse nulle des statistiques de test de chaque étape conditionnellement à ce qui a été observé jusque-là préserve $\alpha$ de manière globale, pourvu que les statistiques de test soient combinées comme il était spécifié au départ. ${ }^{[13,14]}$ Ainsi, il devient possible d'adapter un plan d'expérience dans la mesure où sa modification conserve l'erreur conditionnelle de type I initiale.

Plusieurs méthodes peuvent être utilisées pour combiner l'information apportée par les différentes étapes. Les premiers travaux sur le sujet ont utilisé des tests de combinaison obtenus à partir de la combinaison des degrés de signification (p) obtenus à chaque étape. ${ }^{[13]} \mathrm{D}$ 'autres méthodes reposent sur la fonction d'erreur conditionnelle. ${ }^{[15]}$ Bien que techniquement différentes, toutes ces approches sont liées, et peuvent s'avérer équivalentes dans certains cas. ${ }^{[16]}$

\subsection{Tests multiples}

De nombreux schémas adaptatifs impliquent le test de plusieurs hypothèses nulles comme par exemple le cas dans le schéma de la figure 1. La correction des tests pour contrôler le risque d'erreur de type I global devient alors un point crucial. Prenons le cas d'un essai adaptatif de sélection de dose où, parmi les différentes doses de traitement considérées au départ, une partie seulement sera retenue après l'analyse intermédiaire. On dispose d'un ensemble d'hypothèses nulles correspondant à l'effet de chacune des doses comparativement au bras contrôle. Il faut donc utiliser une méthode qui permette de tester ces hypothèses nulles en contrôlant le risque d'erreur de type I global et en tenant compte 
du fait que des données permettant de tester ces hypothèses ne seront pas nécessairement disponibles à chaque étape, par exemple quand une des doses n'a pas été retenue pour la deuxième étape. Les méthodes utilisées reposent sur le principe des procédures de test fermées, ${ }^{[17]}$ qui contrôle le risque d'erreur de type I $\alpha$ pour l'ensemble des hypothèses testées.

\subsection{Méthodes bayésiennes}

Le principe de l'inférence bayésienne repose sur la combinaison d'une distribution a priori des paramètres d'intérêt et des données recueillies dans l'essai pour obtenir une distribution a posteriori de cette distribution, qui représente le niveau de connaissances actualisées sur ces paramètres. L'utilisation de méthodes bayésiennes n'implique pas nécessairement l'utilisation d'un schéma adaptatif. Néanmoins, l'approche bayésienne est particulièrement adaptée à une démarche séquentielle, dans la mesure où la réactualisation des distributions des paramètres dans un cadre bayésien ne conduit à aucune inflation de risque d'erreur. ${ }^{[18]} \mathrm{Si}$ ce type d'inférence a été plus souvent utilisé pour des essais exploratoires, ${ }^{[19,20]}$ il a été proposé l'utilisation de méthodes bayésiennes pour des essais à visée plus confirmatoire, avec des méthodes pour réévaluer le nombre de sujets ou des essais de phase 2-3. ${ }^{[21,22]}$

\subsection{Pré-spécification, caractéristiques opérationnelles et indépendance de la décision}

Pour les essais confirmatoires, la preuve de l'intégrité de l'essai est capitale et doit être apportée par le promoteur. Plusieurs points sont importants pour garantir cette intégrité.

Le premier point est la pré-spécification de la nature adaptative de l'essai et des adaptations envisagées. D'une part, l'essai doit avoir été planifié comme un essai adaptatif avec un nombre d'adaptations limité. Pour le moins, si une adaptation du plan d'expérience devait être réalisée sans avoir été prévue dès le départ pour faire face à une situation imprévue, il est absolument nécessaire que la décision soit prise avant toute analyse. Le plan d'expérience de la première étape et la règle de combinaison de l'information des différentes étapes doivent être fixés à l'avance, ce qui permet le contrôle strict de l'erreur de type I. De plus, les adaptations qui n'affectent pas directement ce risque d'erreur doivent, autant que possible, être aussi déterminées à l'avance. L'impact des règles de décisions intermédiaires sur l'estimation de l'effet du traitement est alors évalué à l'aide de simulations numériques extensives recouvrant une large gamme de scenarii.

Le second point est le maintien du promoteur en aveugle des résultats de l'essai lors des décisions d'adaptation. Ces décisions, sur la base des résultats de l'essai, doivent être prises par un comité indépendant, dont le rôle et les relations avec le promoteur seront détaillés plus loin.

\section{Schémas adaptatifs pour des essais exploratoires}

\subsection{Essais de phase 1 de recherche de dose}

Les essais de phase 1 ont pour objectif de déterminer la dose maximale tolérée d'un nouveau traitement. En cancérologie, ils ont la particularité d'être conduits chez le sujet malade, du fait de la sévérité de la toxicité potentielle des traitements. Le schéma classique de ces essais, dit « $3+3 »$, consiste à attribuer séquentiellement les doses de traitement prévues à des cohortes de trois patients. Une fois trois patients traités à une dose, les trois patients suivants sont traités à la dose supérieure si aucune toxicité n'a été observée et à la même dose si une toxicité a été observée. Si deux toxicités ou plus sont observées pour une même dose de traitement, l'essai est arrêté.

Un schéma séquentiel alternatif pour la conduite et l'analyse de tels essais, la méthode de réévaluation séquentielle (Continual reassessment method ou CRM), a été proposé. ${ }^{[19]}$ Il repose sur une modélisation paramétrique de la relation dose-toxicité avec une attribution de dose et une estimation séquentielle du paramètre du modèle, jusqu'à l'inclusion d'un nombre fixe préétabli de sujets. Cette méthode a donné lieu à de nombreux travaux qui ont permis d'en corriger la plupart des limites, et aujourd'hui la majorité des applications utilisent la CRM modifiée. ${ }^{[23,24]}$ L'inférence dans ce type de schéma peut être bayésienne ou fréquentiste. Bien que ce schéma ait été développé plus spécifiquement pour les essais de cancérologie, il a aussi été appliqué à d'autres domaines comme la pédiatrie, par exemple.

Les avantages de la CRM modifiée par rapport au schéma $« 3+3 »$ sont une meilleure estimation de la dose maximale tolérée, avec une attribution plus rapide de la bonne dose. Elle permet aussi d'exposer un plus faible nombre de patients à des doses trop toxiques ou inefficaces. En revanche, cette méthode s'avère assez sensible à une toxicité observée à la première dose, en particulier si l'inférence est bayésienne. L'utilisation de ce type de schémas peut être recommandée.

Un exemple d'utilisation de la CRM modifiée concerne la recherche de la dose maximale tolérée de l'administration d'homoharringtonine par voie sous-cutanée chez des patients atteints de leucémie myéloïde aigue. ${ }^{[25]}$ Dans cet essai, cinq niveaux de dose étaient prévus au départ, et la dose maximale tolérée (5 mg. $\mathrm{m}^{-2} /$ jour soit le quatrième niveau de dose) a été définie après l'inclusion de 18 patients évaluables par cohortes de trois. 
Parmi ces patients, 12 ont cependant reçu la dose qui a été finalement retenue, soit deux fois plus que le nombre de patients qui l'auraient reçue avec un schéma classique.

\subsection{Essais intégrés de phases $1-2$}

Des essais de recherche de dose utilisant conjointement des critères de tolérance et d'efficacité ont aussi été proposés, que l'on peut désigner comme des essais de phases 1-2 combinées. ${ }^{[22,26]}$ Ces essais ressemblent dans leur méthodologie générale aux schémas de recherche de dose des essais de phase 1 décrits plus haut.

L'avantage de ces schémas repose sur l'évaluation conjointe de la tolérance et de l'efficacité pour sélectionner la dose, le risque d'éliminer des doses intéressantes étant diminué par rapport aux méthodes traditionnelles. En revanche, ce type d'essais nécessite l'obtention de la réponse en termes d'efficacité pour pouvoir déterminer la dose à attribuer à la prochaine cohorte de patients. En cancérologie, par exemple, la tolérance est évaluée dans un délai de l'ordre d'un mois, mais l'efficacité n'est que rarement évaluée avant trois ou quatre mois, avec pour conséquence un allongement considérable du délai pour définir la bonne dose. De plus, cette approche limite le recul nécessaire pour voir apparaître des toxicités tardives et diminue la connaissance de la tolérance à moyen terme par rapport à un développement en phases séparées.

Ce dernier point a été jugé crucial, et il a été considéré que ce type de schémas était plutôt inapproprié dans le cadre d'une molécule peu connue, ou d'une association de molécules. S'il doit être utilisé, il est plutôt recommandé pour une monothérapie.

\subsection{Essais de phase 2}

Les essais de phase 2 sont des essais exploratoires de l'efficacité d'un traitement. Selon le domaine, l'état des connaissances ou le nombre de molécules à explorer pour la pathologie, de nombreux schémas adaptatifs répondant à différentes questions et contraintes ont pu être utilisés. Les questions peuvent par exemple concerner une recherche de dose ou la priorisation entre plusieurs traitements. Les essais de recherche de dose adaptatifs où le ratio d'allocation des patients entre les différentes doses est adapté en fonction des réponses observées sont notamment assez répandus. ${ }^{[4,20,22]}$

Ce type de schémas présente plusieurs avantages. Ils permettent par exemple d'explorer un plus grand nombre de doses ou d'inclure un plus faible nombre de patients. Ils permettent aussi de traiter plus de patients à la dose qui sera retenue. Ce type de schéma présente cependant certaines limites comme la nécessité d'une logistique lourde, et une certaine complexité méthodologique. Enfin, ils ne sont pas applicables si le critère de réponse est évalué tardivement au regard du rythme du recrutement dans l'essai. Il a néanmoins été jugé par la Table Ronde que l'utilisation de ces méthodes quand cela était possible pouvait être recommandé.

\section{Schémas adaptatifs pour des essais confirmatoires}

\section{1. Essais de phases 2(b)-3 combinées}

L'un des schémas adaptatifs qui a donné lieu à de nombreux travaux ${ }^{[1,5,27,28]}$ et qui a été considéré comme l'un des plus prometteurs $^{[2]}$ est le schéma combiné de phases 2-3 (seamless phase 2-3 design). Ce schéma combine dans un seul essai deux phases de développement qui sont traditionnellement réalisées dans des essais séparés. La première étape est en général utilisée pour sélectionner une ou plusieurs doses de traitement qui seront retenues pour la deuxième étape. L'analyse finale porte alors sur les patients inclus aux deux étapes, avec une méthodologie permettant de garantir la validité des conclusions (voir plus haut).

Par rapport à un développement en deux essais séparés, l'un de phase 2, l'autre de phase 3, ce type de schéma peut présenter plusieurs avantages. En n'interrompant pas le recrutement, il permet un gain de temps global, accentué par un rythme soutenu des inclusions tout au long de l'essai. Il permet aussi un gain en termes de suivi des patients, puisque les patients de la première étape «phase 2 » font aussi partie de l'analyse d'efficacité de la deuxième étape («phase $3 »)$. On peut aussi considérer que ce type de schéma permet de lever une incertitude, par exemple sur la dose ou la variabilité du critère d'évaluation, tout en s'inscrivant dans une démarche d'essai confirmatoire. Enfin, ce schéma permet de compléter les données de tolérance à différentes doses. Notons cependant que, le manque de recul entre les deux phases peut être vu comme un inconvénient.

Le prix à payer pour cette flexibilité et ce gain de temps est une logistique très lourde. Si la planification approfondie de l'essai à partir de simulations extensives de scenarii cliniques est un gage de planification soignée, elle nécessite beaucoup de réflexion et de temps en amont de l'essai. L'utilisation de ce type de schémas nécessite des compétences scientifiques et des négociations avec les autorités compétentes. Des essais avec ce type de schémas sont cependant considérés comme acceptables au cas par cas par ces autorités, si le caractère adaptatif et les adaptations sont pré-spécifiés, si la méthodologie est rigoureuse et si le choix de l'approche est bien étayé. Voulu comme un essai confirmatoire, un essai de phases 2-3 combinées va aussi nécessiter de disposer dès le début de la partie «phase 2 » des prérequis précliniques, et de formes galéniques définitives pour toutes les doses de traitement envisagées. Sa méthodologie doit reposer sur un critère de 
substitution valide pour pouvoir prendre des décisions appropriées quant au choix des doses à garder lors de l'analyse intermédiaire.

Ce type de schémas se justifie donc si le promoteur a une bonne raison d'accélérer le processus de développement, par exemple en cas de besoin thérapeutique important. Il faut de plus noter que si la durée est raccourcie, il n'y a pas nécessairement de gain sur le nombre de sujets à inclure. Enfin, ce type de schémas n'est pas adapté à des situations avec trop d'inconnues ou d'incertitudes : un essai dans lequel trop de paramètres seraient adaptés a de grandes chances de ne pas être considéré comme confirmatoire.

L'exemple présenté figure 1, utilise ce type de schémas pour les essais de phase 2-3 combinées. ${ }^{[29]}$ Cette étude, à visée confirmatoire, a en effet été réalisée en deux étapes combinées utilisant un schéma adaptatif. Au cours de la première étape les patients ont reçu de façon aléatoire, en double aveugle : 4 doses d'indacatérol 75, 150, 300 or $600 \mu \mathrm{g} / \mathrm{j}$, le formoterol (12 $\mu \mathrm{g}$ deux fois par jour) ou un placebo, ou en ouvert, le tiotropium $(18 \mu \mathrm{g} / \mathrm{j})$. Une analyse intermédiaire planifiée à la fin de la première étape a permis à un comité indépendant de sélectionner deux doses d'indacatérol sur la base des données d'efficacité et de tolérance accumulées au cours des deux premières semaines en utilisant des critères d'efficacité prédéfinis. Pendant la seconde période, les deux doses d'indacatérol retenues ont été comparées pendant 26 semaines au tiotropium et au placebo.

\subsection{Réévaluation du nombre de sujets nécessaire}

Des méthodes de réévaluation du nombre de sujets dites en aveugle sont déjà utilisées et ne posent pas de problèmes particuliers en termes d'acceptabilité. Au contraire, des méthodes de réévaluation du nombre de sujets nécessaire dites avec levée d'aveugle ${ }^{[30]}$ ont fait l'objet de fortes controverses dans la littérature qui se sont prolongées lors des débats de la Table Ronde. Avec ces méthodes, les paramètres de nuisance mais aussi parfois la différence d'efficacité à mettre en évidence peuvent être révisés sur la base des données observées lors d'une analyse intermédiaire. La critique principale de ces méthodes repose sur leur manque d'efficience par rapport à un essai séquentiel par groupes. ${ }^{[31,32]}$ Dans le cas d'une réévaluation du nombre de sujets, l'essai est dimensionné au départ avec un effectif assez faible, avec la possibilité d'une augmentation de cet effectif si les résultats sont prometteurs. À l'inverse, un essai séquentiel par groupe planifie dès le départ un nombre plus important de sujets à inclure mais permet d'arrêter précocement le recrutement des sujets. Par ailleurs, il a été noté que les estimateurs de la variance ou de l'effet du traitement lors des premières analyses intermédiaires étaient peu stables, ce qui pouvait entraîner de très fortes inflations du nombre de sujets et le risque de modifier à tort un essai correctement planifié au départ. ${ }^{[33,34]}$ Ce type d'essai adaptatif n'a donc pas été recommandé de manière générale, bien qu'il existe des exemples d'essais acceptés par les autorités compétentes.

\subsection{Sélection d'un sous-groupe}

La détermination d'un sous-groupe de patients pour lesquels le traitement évalué est efficace peut s'avérer importante. Si l'on dispose d'un marqueur biologique qui peut être associé à l'efficacité du traitement, des schémas adaptatifs, dits schémas d'enrichissement, ont été proposés. ${ }^{[3,36]}$ Ils permettent de débuter l'essai dans la population générale, avec la possibilité de restreindre les inclusions à un sous-groupe après une analyse intermédiaire si les résultats laissent supposer que le traitement n'est efficace que dans ce sous-groupe.

Ce type de schéma ne soulève pas de difficultés particulières si le marqueur définissant les sous-groupes est spécifié à l'avance. En revanche, si ce marqueur n'a pas été défini à l'avance, il est nécessaire que la justification de son utilisation soit complètement extérieure à l'essai. Il semble aussi utile de recommander que la méthodologie d'adaptation soit déterminée avant toute analyse des données de l'essai.

\section{Rôle du comité indépendant et des intervenants}

\subsection{Comité indépendant}

Afin de garantir l'intégrité de l'essai, les adaptations du plan d'expérience lors les analyses intermédiaires doivent être conseillées par un comité indépendant du promoteur, celui-ci ne devant pas avoir accès aux données. Du fait de la possibilité d'adapter le plan d'expérience, le comité indépendant a un rôle accru par rapport à des essais plus classiques, où il surveille généralement la tolérance. Pour maintenir l'intégrité des résultats, il est primordial que les circuits d'information soient sécurisés pour prévenir tout soupçon de levée d'insu. En particulier, un statisticien indépendant du promoteur et du comité indépendant est parfois impliqué dans certains schémas adaptatifs. Ce statisticien, à l'interface entre ces derniers, est chargé de réaliser les analyses nécessaires au comité indépendant.

En soi, le comité indépendant ne décide pas des adaptations, mais il émet des recommandations sur la base des résultats de l'essai, que ce soient des données d'efficacité ou de tolérance, mais aussi de toute source de données extérieures à l'essai qu'il jugerait utile de prendre en compte. Les décisions d'adaptation doivent cependant répondre aux stratégies de développement du promoteur. Or le promoteur ne peut pas examiner les données sur lesquelles les décisions d'adaptation s'appuient. Il est donc recommandé 
qu'il remette une charte au comité indépendant, dans laquelle il présente les scenarii envisagés au départ et les décisions qu'il souhaite prendre en fonction des résultats intermédiaires. Malgré cela, il semble nécessaire qu'une part de décision soit déléguée au comité indépendant, notamment pour faire face à des situations non prévues dans la charte.

Pour faire face à ces situations inattendues, certains ont envisagé une intervention limitée de représentants du promoteur non directement impliqués dans l'étude dans les décisions du comité indépendant. ${ }^{[3]}$ À notre connaissance au moins un essai a eu recours à cette stratégie. Cependant, il n'existe aucune jurisprudence et le risque de requalification de l'essai en « exploratoire » est trop important pour le recommander.

\subsection{Relations avec les autorités compétentes}

Les autorités compétentes apportent une attention particulière aux essais confirmatoires conduits avec des schémas adaptatifs et des interactions particulières entre le promoteur et ces autorités sont attendues. En amont de l'essai, le côté adaptatif peut être discuté et il est conseillé au promoteur de demander un avis scientifique aux autorités d'enregistrement. On peut noter par exemple à ce sujet que la FDA a rendu des avis non contraignants (non binding) sur des schémas adaptatifs ayant par définition une méthodologie innovante. Les autorités sont aussi destinataires de la charte que le promoteur remet au comité indépendant et sont attentives au respect des recommandations de ce comité en cours d'essai. Si le promoteur passait outre ces recommandations, il est probable que les autorités compétentes saisissent le comité indépendant, et éventuellement suspendent l'essai de façon conservatoire.

\section{Contraintes : logistique et risques}

Nous avons vu que les schémas adaptatifs induisent des contraintes, en particulier d'un point de vue logistique. Par ailleurs, il paraît important d'évaluer les risques associés à l'utilisation de tels schémas en regard des avantages qu'ils peuvent apporter.

\section{1. Contraintes et logistique}

Les schémas adaptatifs nécessitent en général une planification plus approfondie que les essais classiques. Par exemple, les essais précoces décrits au paragraphe 3.1 reposent sur un modèle paramétrique de la relation dose toxicité qu'il faut déterminer et justifier à l'avance. Pour les essais de phase 2-3 décrits au paragraphe 4.1, l'exigence de pré-planification contraint à envisager à l'avance un grand nombre de scenarii et les adaptations correspondantes. Dans de nombreux cas, les caractéristiques opérationnelles des schémas utilisés sont évaluées a priori par des simulations numériques, dans la mesure où elles ne sont pas connues analytiquement à l'avance.

Ces schémas entraînent aussi des réunions de concertation plus larges et plus nombreuses, impliquant parfois des intervenants plus en amont que d'habitude. Par exemple, il peut s'avérer nécessaire de rencontrer les représentants des autorités compétentes, et d'impliquer plus les membres des comités de l'essai (comité scientifique, comité indépendant...) Dans un essai de phase 2-3, les responsables de la galénique seront impliqués bien plus tôt que dans un développement classique. Enfin, il faut former les équipes internes et les prestataires, ainsi que les investigateurs à la méthodologie de l'essai, qui ne leur est pas familière.

En termes de logistique des produits, il faut parfois que la forme commerciale soit disponible plus tôt, comme envisagé précédemment. Un plus grand nombre de doses testées dans un essai de phase 2-3 peut aussi nécessiter le développement de formes galéniques «flexibles », comme des mini-comprimés, par exemple. La flexibilité du plan d'expérience a aussi un impact sur les circuits d'approvisionnement et de gestion des stocks.

Enfin, des contraintes pèsent sur la gestion des données de l'essai qui doivent être à la fois sécurisées et valides. Des données valides doivent être disponibles rapidement pour ne pas retarder les décisions d'adaptation qui pourraient être prises. Il est donc nécessaire de monitorer les données en continu. Enfin, les flux de données doivent être sécurisés, comme rappelé plus haut, ce qui induit aussi de nouvelles contraintes.

\subsection{Risques}

Plusieurs types de risques peuvent être envisagés. Pour l'essai, et donc pour le promoteur, le risque le plus important est celui d'une mise en jeu de la validité et de l'intégrité des résultats, qui conduirait à une requalification de l'essai de confirmatoire en exploratoire par les autorités compétentes.

En ce qui concerne ces autorités, on peut noter une certaine réticence à autoriser un essai sans avoir les données de la phase précédente, par exemple un essai de phase 3 sans résultats de phase 2 ou un essai de phase 2 sans résultats de phase 1 . Cette réticence repose sur la crainte d'un manque de recul entre les phases entraînant une perte d'information sur la tolérance à moyen terme.

Pour les patients, hormis ce possible manque de recul dans les essais de phase 1-2 ou 2-3 combinées, il semble au contraire que les risques soient moindres dans les essais de phase 1 ou 2 adaptatifs, puisque ces schémas tendent justement à faire en sorte qu'un plus grand nombre de patients soient traités à la meilleure dose possible. Les patients peuvent aussi être suivis plus longtemps 
dans le cadre d'une recherche, et donc mieux suivis, dans des essais de phase 2-3 que dans des essais de phase 2 séparés de la phase 3 .

Enfin, il faut aussi que le promoteur puisse garantir les moyens d'assurer la logistique et de conduire l'essai jusqu'à son terme quelle que soit l'adaptation qui sera décidée à l'analyse intermédiaire.

\section{Conclusion}

Ainsi, comparés aux schémas traditionnels où le plan d'expérience est fixé d'avance, les méthodes adaptatives représentent une approche séduisante d'un point de vue à la fois scientifique et pragmatique. Leur principal intérêt consiste à aboutir plus rapidement voire plus efficacement à l'information attendue. Les diverses situations abordées au cours de cette revue montrent que le recours à ces méthodes, en phase exploratoire ou confirmatoire du développement, est souvent possible et accepté par les autorités compétentes à condition de garantir l'intégrité et la validité de l'essai. Pour ce faire, les contraintes sont nombreuses tant financières que logistiques et il est nécessaire de les anticiper. Dans les essais confirmatoires notamment, le recours à ces méthodes nécessite donc à la fois, rigueur, et anticipation dans la planification comme dans l'exécution, concertation avec les autorités compétentes et présence d'un comité indépendant. Si toutes ces conditions sont respectées, l'utilisation des méthodes adaptatives doit être encouragée.

\section{Participants}

Anne d'Andon (HAS), François Bassompierre (DRCD APHP, Paris), Jean-Michel Béhier (MSD Chibret), Chantal Belorgey (Afssaps), Jacques Bénichou (CHU Rouen), Gilles Berdeaux, Loic Bergougnoux (Roche), Pascal Bilbault (Boehringer Ingelheim), Olivier Chassany (DRCD AP-HP, Paris), Christian Funck Brentano (GH Pitié-Saplêtrière, Paris), Martha Gersberg (Gersberg Consulting), Claire Labreveux (GlaxoSmithKline), Catherine Lassale (LEEM), Céleste Lebbé (Hôpital Saint-Louis, Paris), Brigitte Lecocq (Novartis Pharma S.A.S.), Vincent Lévy (Hôpital Avicenne, Bobigny), François Montestruc (AB Sciences), Caroline Morgan (Cardinal Systems), Gaelle Nachbaur (GlaxoSmithKline), Brigitte Palestro (IPSEN), Xavier Paoletti (Institut Curie, Paris), Raphaël Porcher (Hôpital Saint-Louis, Paris), Anne Raison (Afssaps), Laurent Spiess (Cytel), Nathalie Strub (DNDI, Genève), Christian Vitzling (Novartis Pharma), Muriel Vray (Institut Pasteur, Paris).

\section{Références}

1. Bretz F, Schmidli H, König F, et al. Confirmatory seamless phase II/III clinical trials with hypotheses selection at interim: general concepts. Biom J 2006; 48: 623-34

2. Gallo P, Chuang-Stein C, Dragalin V, et al. Adaptive designs in clinical drug development. An executive summary of the PhRMA Working Group. J Biopharm Stat 2006; 16: 275-83

3. Bretz F, Koenig F, Brannath W, et al. Adaptive designs for confirmatory clinical trials. Stat Med 2009; 28: 1181-217

4. Bretz F, Branson M, Burman CF, et al. Adaptivity in drug discovery and development. Drug Development Research 2009; 70:169-90

5. Schmidli H, Bretz F, Racine A, et al. Confirmatory seamless phase II/III clinical trials with hypotheses selection at interim: applications and practical considerations. Biom J 2006; 48: 635-43

6. FDA. Critical Path Opportunities List. 2006 http://www.fda.gov/oc/ initiatives/criticalpath/

7. CHMP. Reflection paper on methodological issues in confirmatory clinical trials planned with an adaptive design. 2009 http: //www. ema.europa. eu/docs/en_GB/document_library/Scientific_guideline/ 2009/09/WC500003616.pdf

8. FDA. Guidance for Industry: adaptive design clinical trials for drugs and biologics. 2010 http://www.fda.gov/downloads/drugs/ guidancecomplianceregulatoryinformation/guidances/ ucm201790.pdf

9. Dragalin V. Adaptive designs: terminology and classification. Drug Info J 2006; 40: 425-35

10. Brannath W, Koenig F, Bauer P. Multiplicity and flexibility in clinical trials. Pharm Stat 2007; 6: 205-16

11. CPMP. ICH E9: note for guidance on statistical priniples for clinical trials [Internet]. 1998 http://www.ema.europa.eu/docs/ en_GB/document_library/Scientific_guideline/2009/09/ WC500002928.pdf

12. Bauer P, Brannath W, Posch M. Flexible two-stage designs: an overview. Meth Info Med 2001; 40: 117-21

13. Bauer P, Köhne K. Evaluation of experiments with adaptive interim analyses. Biometrics 1994; 50: 1029-41

14. Brannath W, Posch M, Bauer P. Recursive combination tests. J Am Statist Assoc 2002; 97(457): 236-44

15. Proschan MA, Hunsberger SA. Designed extension of studies based on conditional power. Biometrics 1995; 51: 1315-24

16. Posch M, Bauer P. Adaptive two stage designs and the conditional error function. Biom J 1999; 41: 689-96

17. Marcus R, Eric P, Gabriel KR. On closed testing procedures with special reference to ordered analysis of variance. Biometrika 1976; 63: 655-60

18. Spiegelhalter DJ, Abrams KR, Myles JP. Bayesian approaches to clinical trials and health-care evaluation. Chichester: Wiley, 2004

19. O’Quigley J, Pepe M, Fisher L. Continual reassessment method: a practical design for phase 1 clinical trials in cancer. Biometrics 1990; 46: 33-48

20. Berry DA, Mueller P, Grieve AP, et al. Adaptive Bayesian designs for doseranging drug trials. Case Studies in Bayesian Statistics 2001; 5: 99-181

21. Schmidli H, Bretz F, Racine Poon A. Bayesian predictive power for interim adaptation in seamless phase II/III trials where the endpoint is survival up to some specified timepoint. Stat Med 2007; 26: 4925-38

22. Berry SM, Carlin BP, Lee JJ, et al. Bayesian adaptive methods for clinical trials. Boca Raton: CRC Press, 2010

23. Garrett-Mayer E. The continual reassessment method for dose-finding studies: a tutorial. Clin Trials 2006; 3: 57-71

24. O'Quigley J, Zohar S. Experimental designs for phase I and phase I/II dosefinding studies. Br J Cancer 2006; 94: 609-13 
25. Levy V, Zohar S, Bardin C, et al. A phase I dose-finding and pharmacokinetic study of subcutaneous semisynthetic homoharringtonine (ssHHT) in patients with advanced acute myeloid leukaemia. Br J Cancer 2006; 95: 253-9

26. Huang X, Biswas S, Oki Y, et al. A parallel phase I/II clinical trial design for combination therapies. Biometrics 2007; 63: 429-36

27. Posch M, Koenig F, Branson M, et al. Testing and estimation in flexible group sequential designs with adaptive treatment selection. Stat Med 2005; 24: 3697-714

28. Liu Q, Pledger GW. Phase 2 and 3 combination designs to accelerate drug development. J Am Statist Assoc 2005; 100(470): 493-502

29. Donohue JF, Fogarty C, Lotvall J, et al. Once-daily bronchodilators for chronic obstructive pulmonary disease: indacaterol versus tiotropium. Am J Resp Crit Care Med 2010; 182: 155-62

30. Chuang-Stein C, Anderson K, Gallo P, et al. Sample size re-estimation: a review and recommendations. Drug Info J 2006; 40: 475-84

31. Tsiatis AA, Mehta C. On the inefficiency of the adaptive design for monitoring clinical trials. Biometrika 2003; 90: 367-78
32. Burman CF, Sonesson C. Are flexible designs sound? Biometrics 2006; 62: 664-9

33. Bauer P, Koenig $\mathrm{F}$. The reassessment of trial perspectives from interim data-a critical view. Stat Med 2006; 25: 23-36

34. Desseaux K, Porcher R. Flexible two-stage design with sample size reassessment for survival trials. Stat Med 2007; 26: 5002-13

35. Wang SJ, O’Neill RT, Hung HM. Approaches to evaluation of treatment effect in randomized clinical trials with genomic subset. Pharm Stat 2007; 6: 227-44

36. Brannath W, Zuber E, Branson M, et al. Confirmatory adaptive designs with Bayesian decision tools for a targeted therapy in oncology. Stat Med 2009; 28: $1445-63$

Correspondance et offprints : Brigitte Lecocq, Laboratoire Novartis, 2-4 rue Lionel Terray, 92506 Rueil Malmaison cedex, France.

E-mail : brigitte.lecocq@novartis.com 